\title{
Vers une description du mécanisme d'assemblage du fuseau mitotique à l'échelle moléculaire
}

L'assemblage du fuseau mitotique, prélude à la mitose, nécessite une réorganisation du réseau de microtubules déclenchée par le complexe cycline $\mathrm{B} / \mathrm{p} 34^{\text {cdc2 }}$ et impliquant l'intervention de moteurs moléculaires de type kinésine. Ce type de molécule, ainsi que d'autres moteurs de la famille des dynéines, interviennent aussi pour assurer le mouvement des chromosomes le long des microtubules. Tous ces phénomènes pourraient être finement contrôlés par l'équilibre de réactions de phosphorylation (catalysées par des protéine kinases) et de déphosphorylation (catalysées par des protéine phosphatases).

\section{Éric Karsenti}

E. Karsenti : chef de groupe. Laboratoire curopéen de biologie moléculaire (EMBL), département de biologie cellulaire, Meyerhofstrasse 1, D-6900, Heidelberg, Allemagne. ors de la division cellulaire, la distribution des chromatides aux cellules filles est effectuée par un appareil complexe qui a fasciné des générations de biologistes par son esthétisme : le fuseau mitotique (figure 1, p. 132). L'attrait un peu mystique qu'a produit cet objet est probablement dû à sa structure, à la fois géométrique, parfaitement bipolaire, et à sa dynamique. En effet, le fuseau mitotique est une structure évanescente, elle apparaît pendant quelques minutes pour remplir sa fonction lors de la division cellulaire, puis disparaît. Pour tenter de comprendre en termes rationnels la nature du fuseau, il est nécessaire de décrire ses composants essentiels, comment ils interagissent entre eux, comment ces interactions sont gouvernées dans le temps et dans l'espace, et finalement comment les propriétés intrinsèques des nombreuses molécules qui composent le fuseau peuvent rendre compte de sa fonction globale. Au cours des dix dernières années, la direction à suivre pour aborder ce problème est progressivement apparue. Cette approche implique la manipulation de technologies très variées mais aussi l'utilisation de concepts appartenant aussi bien à la biologie moléculaire qu'à la physique. L'étude du fuseau mitotique est un exemple d'étude de la morphogenèse cellulaire et il est certain que les méthodes et les concepts développés dans ce domaine auront des applications très générales.

Les structures cellulaires essentielles du fuseau mitotique sont les microtubules. Ces tubes interagissent avec 


\section{RÉFÉRENCES}

1. Alberts B, Bray I), Lewis J, Raff M, Roberts K, Watson JI). Biologie moléculaire de la cellule. Paris : Flammarion, 1990 : 645-62.

2. Carlier MF. Nucleotide hydrolysis in cytoskeletal assembly. Curr Op Cell Biol $1991 ; 3$ : 12-7.

3. Kirschner M, Mitchison T. Beyond selfassembly: from microtubules to morphogenesis. Cell $1986 ; 45$ : 329-42.

4. Walker RA, O'Brien ET, Pryer NK, et al. Dynamic instability of individual microtubules analyzed by video light microscopy : rate constants and transition frequencies. $J$ Cell Biol 1988 ; 107 : 1437-48.

5. Vale RI), Goldstein LSB. One motor, many tails : an expanding repertoire of force-generating enzymes. Cell $1990 ; 60$ : 883-5.

6. Mcloonald HB, Stewart RJ, Goldstein LS. The kinesin-like ncd protein of Irosophila is a minus end-directed microtubule motor. Cell $1990 ; 63$ : 1159-65.

7. Bucndia B, Draetta G, Karsenti E Regulation of the microtubule nucleating activity of centrosomes in Xenopus egg extracts : rolc of cyclin $\mathrm{A}$ associated protein kinasc. J Cell Biol 1992 ; 116 : 1431-42.

8. Rieder CI, Alexander SP. Kinetochores are transported poleward along a single astral microtubule during chromosome attachment to the spindle in new lung cells. J Cell Biol $1990 ; 110$ : 81-95.

9. Merdes A, De Mey J. The mechanism of kinetochore-spindle attachment and polewards movement analyzed in PtK2 cells at the prophase-prometaphase transition. Eur J Cell Biol 1990 ; 53 : 313-25.

10. Sawin KE, Scholey JM. Motor proteins in cell division. Trends Cell Biol 1991; 1 122-9.

11. Cassimeris LU, Walker RA, Pryer NK, Salmon EI). Dynamic instability of microtubules. Bioassays $1987 ; 7: 149-54$.

12. Lohka M, Maller J. Induction of nuclear cnvelope breakdown chromosome condensation, and spindle formation in cellfree extracts. $J$ Cell Biol 1985 ; 101 : 518-23.

13. Murray A. Cell cycle extracts. In : Kay BK, Peng HB, eds. Xenopus laevis : Practical Uses in Cell and Molecular Biology, San DiegoNew York-Boston-London-Sydney-TokyoToronto : Academic press, 1991 : 581-605.

14. Newport J. Nuclear reconstitution in vitro : stages of assembly around protein-free I)NA. Cell $1987 ; 48: 205-17$.

15. Verde F, Labbé JC, Dorée M, Karsenti E. Regulation of microtubule dynamics by cdc2 protein kinase in cell-free extracts of Xenopus eggs. Nature $1990 ; 343$ : 233-8.

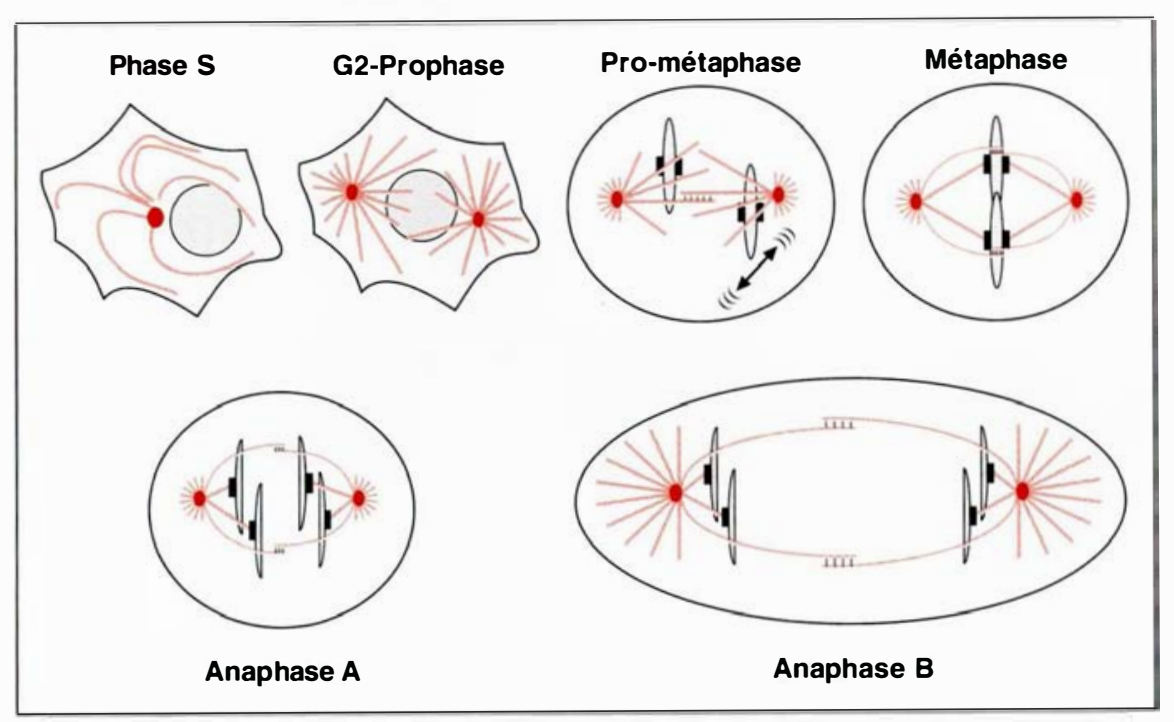

Figure 1. Représentation schématique des différentes phases de la mitose. Le centrosome est coloré en rouge, les microtubules en rose, les kinétochores en noir et les interdigitations entre microtubules dans la région équatoriale en gris

les chromosomes au niveau de régions spécialisées, les kinétochores. Ils servent de guide pour l'alignement des chromosomes sur la plaque métaphasique puis pour la migration des chromatides vers les deux cellules filles (figure 1). Pour découvrir les principes qui gouvernent l'assemblage du fuseau mitotique, il est donc nécessaire de répondre aux questions suivantes : (1) quelles sont la composition, la structure et les propriétés dynamiques des microtubules; (2) quelles sont les molécules qui gouvernent l'organisation supra-moléculaire des microtubules en un fuseau; (3) comment les microtubules interagissent-ils avec les chromosomes ; (4) comment les chromosomes se déplacent-ils le long des microtubules et (5) quels sont les signaux qui déterminent quand le fuseau doit s'assembler, puis fonctionner, c'est-àdire distribuer les chromatides aux deux cellules filles.

\section{Les microtubules}

Les microtubules sont des tubes creux composés de protofilaments (souvent 13) formés par l'assemblage d'hétérodimères de tubuline (appelés $\alpha$ et $\beta$, figure $2 a$ ). Ces tubes ont des propriétés remarquables qui sont dues à la nature des sous-unités de tubuline. Le dimère de tubuline présente deux sites de liaison pour le GTP, un site échangeable et un site non échangeable, et il est asymétrique. Cette asymétrie se retrouve dans le microtubule, qui est une structure orientée. Une extrémité a été appelée + (plus) et l'autre - (moins) (figure 2a) [1]

\section{Hydrolyse du GTP et instabilité dynamique}

Lorsque la tubuline polymérise, les molécules de GTP liées au site échangeable sont hydrolysées. Il y a donc consommation d'énergie associée à l'assemblage des microtubules. La présence de GTP sur les sous-unités de tubuline est nécessaire à la polymérisation, mais l'hydrolyse ne l'est pas. En fait, lorsque le microtubule polymérise, il existe un temps de latence très court entre l'association d'une molécule de tubuline à l'extrémité du microtubule et l'hydrolyse du GTP. Si bien que le corps du microtubule est composé de tubuline GDP alors que son extrémité est composée de tubuline GTP (figure 2a). Ce phénomène est très important parce que les interactions entre les molécules de tubuline GDP au sein du microtubule sont beaucoup plus faibles que les 
interactions entre les molécules de tubuline GTP qui viennent de s'associer au microtubule [2]. Par conséquent, si par hasard le GTP présent dans les molécules de tubuline qui constituent l'extrémité d'un microtubule en cours d'assemblage est hydrolysé, cette extrémité devient ins- table et le microtubule dépolymérise. La conséquence de ce phénomène biochimique est une propriété importante des microtubules: l'instabilité dynamique [3]. Ce terme signifie qu'un microtubule en cours de polymérisation a toujours une certaine probabilité de stopper brutalement son assemblage et d'entrer dans une phase de dépolymérisation. L'instabilité est dite dynamique car associée à la croissance du microtubule. Les propriétés dynamiques d'une population de microtubules peuvent donc être décrites par quatre paramètres : leur vitesse d'élongation $\left(V_{c}\right)$, leur

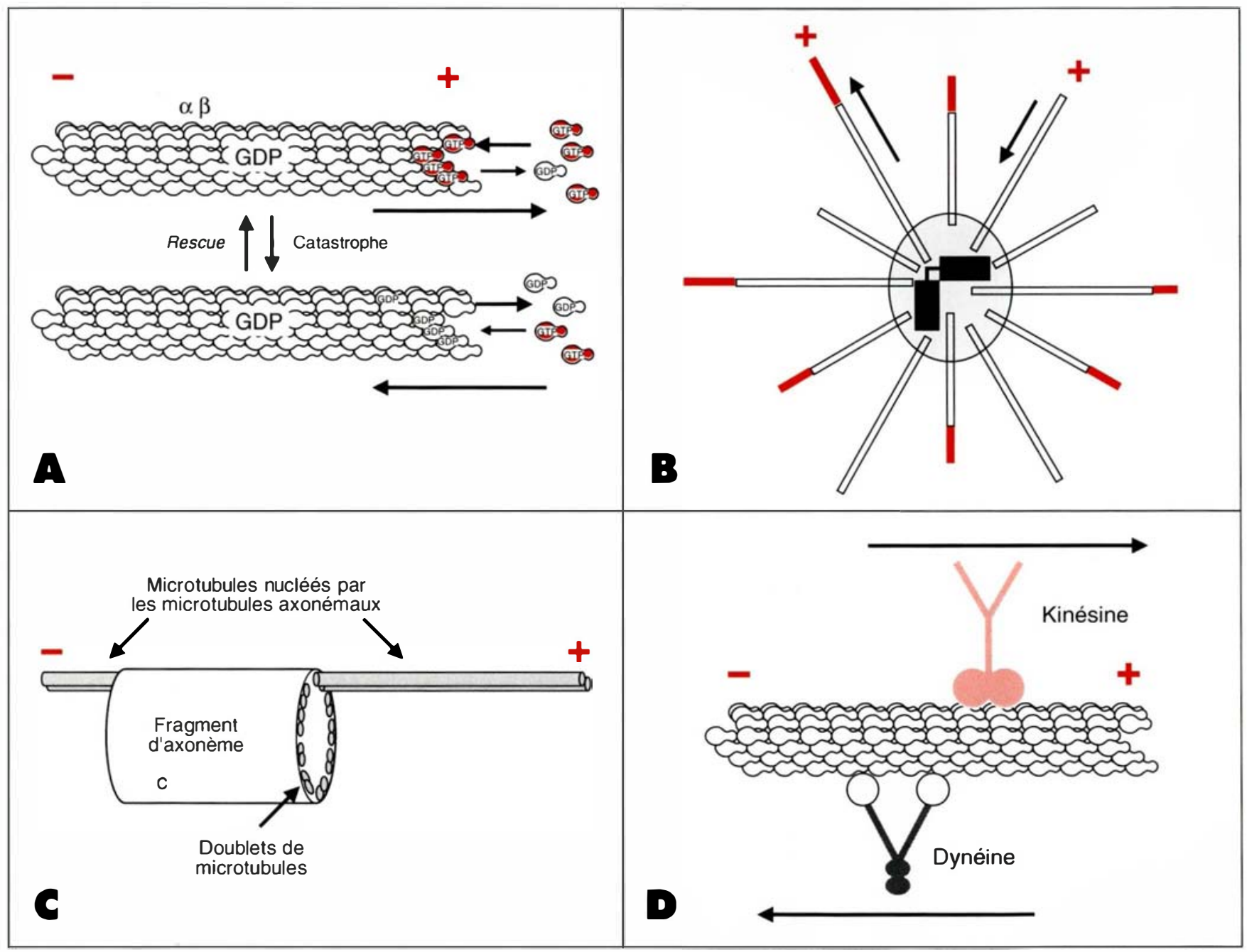

Figure 2. A. Structure du microtubule et rôle du GTP dans l'assemblage. Les dimères de tubuline sont symbolisés en blanc $(\alpha)$ et gris $(\beta)$. Le microtubule du haut est en phase d'élongation, son extrémité + contient des molécules de "tubuline GTP". Celui du bas en phase de dépolymérisation. Son extrémité + contient de la tubuline GDP. Le comportement de l'extrémité - n'est pas examiné ici. B. Nucléation des microtubules par un centrosome. Ici, un centrosome isolé (centriole en noir entouré de son matériel de nucléation des microtubules, très schématisé) est incubé dans une solution de dimères de tubuline dans des conditions où l'initiation de l'assemblage ne se produit pas spontanément. Seuls les microtubules nucléés par le centrosome polymérisent, mais ils sont instables. Ceux qui sont en phase d'élongation ont un "cap GTP" (en rouge), les autres sont en phase de dépolymérisation. Tous les microtubules sont nucléés avec leur extrémité plus distale par rapport au centrosome. C. Polarité d'assemblage des microtubules. Ici, un fragment d'axonème (composé de 9 doublets de microtubules) est incubé dans de la tubuline pure (comme le centrosome). Les microtubules de l'axonème ont leurs deux extrémités libres et les dimères de tubuline polymérisent au deux extrémités. Cependant, la vitesse d'assemblage est plus faible à l'extrémité -. Par conséquent, les microtubules nucléés par cette extrémité sont toujours plus courts. Cette méthode permet d'étudier le comportement des deux extrémités des microtubules en vidéo-microscopie. D. Polarité fonctionnelle des microtubules. En général, mais non systématiquement (voir texte), les molécules de type kinésine se déplacent vers l'extrémité + des microtubules et les molécules de dynéine vers l'extrémité - 


\section{RÉFÉRENCES}

16. Belmont LD, Hyman AA, Sawin KE, Mitchison TJ. Real-time visualization of cell cycle-dependent changes in microtubule dynamics in cytoplasmic extracts. Cell 1990 ; 62 : 579-89.

17. Verde F, Dogterom M, Stelzer E, Karsenti E, Leibler S. Control of microtubule dynamics and length by cyclin $\mathrm{A}$ and cyclin B dependent kinases in Xenopus egg extracts. J Cell Biol $1992 ; 118: 132-7$.

18. Dorće M. Control for M-phase by maturation promoting factor. Curr Op Cell Biol $1990 ; 2$ : 269-73.

19. Murray AW, Kirschner MW. Cyclin synthesis drives the carly embryonic cell cycle. Nature $1989 ; 339$ : 275-80.

20. Félix MA, Labbé JC, Dorće M, Hunt $\mathrm{T}, \mathrm{K}$ arsenti $\mathrm{E}$. Triggering of cyclin degradation in interphase extracts of amphibian eggs by cdc2 kinase. Nature $1990 ; 346$ : 379-82.

21. Karsenti E, Verde F, Félix MA. Role of type 1 and type 2A protein phosphatases in the cell cycle. Adv Prot Phosph 1991; $6: 453-82$

22. Leeiss D, Félix MA, Karsenti E. Association of cyclin-bound p34cdc2 with subcellular structures in Xenopus eggs. $J$ Cell Sci $1992 ; 102$ : 285-97.

23. Labbć JC, Capony JP, Caput D, et al. MPF from starfish oocytes at first meiotic metaphase is a heterodimer containing one molecule of cdc2 and one molecule of cyclin B. $E M B O J 1989 ; 8$ : 3053-8

24. Solomon MJ, Glotzer M, Lee TH, Philippe M, Kirschner MW. Cyclin activation of p34cdc2. Cell 1990; 63 : 1013-24.

25. Clarke P, Leiss D, Pagano M, Karsenti E. Cyclin A- and cyclin B-dependent kin ase are regulated by different mechanisms in Xenopus egg extracts. EMBO J $1992 ; 11$ : 1751-61

26. Sawin KE, Mitchison TJ. Mitotic spindle assembly by two different pathways in vitro. J Cell Biol 1991; 112: 925-40.

27. Mitchison TJ. Microtubule dynamics and kinetochore function in mitosis. Annu Rev Cell Biol 1988 ; 4 : 527-49.

28. Karsenti E, Newport J, Hubble R, Kirschner M. Interconversion of metaphase and interphase microtubule arrays, as studied by the injection of centrosomes and nuclei into Xenopus eggs. J Cell Biol 1984 ; 98 : $1730-45$.

29. Nicklas RB, Kubai DF. Microtubules, chromosome movement, and reorientation after chromosomes are detached from the spindle by micromanipulation. Chomosome $1985 ; 92: 313-24$. vitesse de décroissance $\left(V_{d}\right)$, la fréquence avec laquelle ils transitent de la phase d'élongation à la phase de décroissance (appelée catastrophe, $f_{\text {cat }}$ ) et la fréquence inverse (appelée rescue, $\mathrm{f}_{\mathrm{res}}$ ).

Dans la cellule, les conditions sont souvent telles que les microtubules ne polymérisent pas spontanément. Leur assemblage est catalysé par un centre de nucléation, généralement un centrosome (figure $2 b$ ). Le centrosome est une structure composée de deux cyclindres centriolaires entourés d'un " nuage " de composition encore mal définie. Ce nuage contient les molécules capables de catalyser l'assemblage des microtubules. Du fait de l'instabilité dynamique, les microtubules nucléés par les centrosomes ne se comportent pas tous de la même façon. A un instant donné, certains sont en phase d'élongation (ceux qui possèdent un "cap " de tubuline GTP à leur extrémité) alors que d'autres dépolymérisent. La figure $2 b$ illustre ce processus en prenant comme exemple ce qui se passe in vitro lorsque l'on ajoutc des centrosomes isolés à une préparation de tubuline purifiée à partir de cerveau de bœuf.

In vivo, une situation similaire peut être observée à des degrés divers selon les cellules. En effet, l'instabilité dynamique est modulée par la présence de protéines capables de s'associer aux microtubules, les MAP (microtubule associated proteins). $\mathrm{La}$ nature des MAP et leur effet sur la dynamique des microtubules dépendent du type cellulaire.

\section{La polarité des microtubules}

La polarité structurale des microtubules a deux conséquences fonctionnelles. L'une concerne leur dynamique, et l'autre leur fonction dans le transport intracellulaire.

L'extrémité + (plus) polymérise plus rapidement que l'extrémité (moins) et est plus dynamique. Les microtubules nucléés par les centrosomes polymérisent toujours avec l'extrémité + distale par rapport au centrosome (figure $2 b$ ). On peut mettre clairement en évidence cette différence entre les deux extrémités en ajoutant des fragments de cils ou flagelles, qui sont composés de microtubules très stables et orientés, à une solution de tubuline. La tubuline polymérise et forme des microtubules aux deux extrémités du flagelle. Mais les microtubules s'allongent beaucoup plus rapidement d'un côté que de l'autre (figure 2c). C'est l'extrémité + $[1,4]$.

L'une des fonctions des microtubules est de permettre le transport de vésiculcs cytoplasmiques en interphase et des chromosomes dans le fuseau mitotique. Ce transport est effectué par des moteurs protéiques qui se déplacent le long des microtubules en utilisant l'hydrolyse de l'ATP comme source d'énergie (figure $2 d$ ), comme la myosine le fait le long des filaments d'actine dans le muscle. Ces moteurs lisent la polarité des microtubules [5]. Certains se déplacent vers l'extrémité + et d'autres vers l'extrémité - . Il existe deux grands types de moteurs, les uns appartiennent à la famille des kinćsines et les autres à la famille des dinéines. En général, mais il semble y avoir des exceptions [6], les kinésines se déplacent vers l'extrémité + et les dinéines vers l'extrémité - . La bipolarité du fuseau se reflète dans le fait que les microtubules qui composent les deux demi-fuseaux ont des polarités opposées. Ils sont nucléés par les centrosomes présents à chaque pôle et ils interagissent entre eux dans la région équatoriale par lcur extrémité + (figure 1). C'est aussi cette extrémité qui interagit avec les kinétochores des chromosomes.

\section{Dynamique des microtubules et assemblage du fuseau}

L'assemblage du fuseau mitotique implique une série d'événements qui se succèdent dans un ordre bien précis (figure 1). Pendant la phase de réplication de l'ADN (phase S), lc centrosome se duplique. Cette duplication est suivie par la séparation des deux centrosomes, qui migrent autour du noyau et finalement se positionnent de façon diamétralement opposée. Pendant cette période (prophase), l'activité de nucléation des centrosomes augmente considérablement [7] et le réseau interphasique de microtubules se désagrège progressivement au profit des deux asters de microtubules prophasiques qui envahissent le cytoplasme. Lorsque l'enveloppe nucléaire se disperse en fin de 
prophase, les microtubules astériens raccourcissent et leur assemblage s'oriente préférentiellement vers les chromosomes en cours de condensation. Certains microtubules entrent en contact avec les kinétochores. Aussitôt, le chromosome correspondant migre à grande vitesse le long du microtubule vers le centrosome, puis s'en éloigne $[8,9]$. Pendant cette période, les chromosomes oscillent entre les deux pôles jusqu'à ce qu'ils trouvent une position d'équilibre définissant la plaque métaphasique. Les chromosomes continuent alors à osciller entre les deux pôles, mais avec une faible amplitude. Lorsque les chromatides se séparent, les chromosomes migrent vers le pôle faisant face au kinétochore correspondant, c'est l'anaphase A. Ce mouvement est accompagné d'un raccourcissement des microtubules kinétochoriaux. Pendant ce mouvement, la longueur des autres microtubules qui interagissent dans la zone équatoriale ne change pas. Ensuite, ils s'allongent, augmentant ainsi la distance entre les deux pôles du fuseau : c'est l'anaphase B. Finalement, la cellule se divise en deux cellules filles.

Cette réorganisation complexe des microtubules doit être coordonnée dans le temps. Par ailleurs, elle est possible du fait des propriétés dynamiques des microtubules exposées cidessus. Enfin, il existe toute une série de mouvements, et des résultats récents ont clairement montré que des moteurs protéiques de type dinéine et kinésine jouent un rôle dans ces mouvements et sont effectivement impliqués dans l'assemblage et la fonction du fuseau mitotique [10].

Le contrôle de la dynamique des microtubules en mitose

Lors de l'assemblage du fuseau mitotique, la dynamique des microtubules change de façon spectaculaire. In vivo, la demi-vie des microtubules est de l'ordre de 10 à 20 minutes dans le réseau interphasique. Elle décroît à environ 30 secondes- 1 minute dans le fuseau mitotique. Dans le cadre de la théorie de l'instabilité dynamique des microtubules, cette observation signifie que les microtubules du fuseau alternent rapidement entre des phases d'élongation et de dépolymé$m / s n^{\circ} 2$ vol. 9, février 93 risation [11]. Il a été difficile de démontrer l'existence de ce phénomène in vivo, parce que la densité en microtubules dans le fuseau rend difficile l'observation de microtubules individuels. Cependant, il a été possible d'aborder cette question in vitro en utilisant du cytoplasme préparé à partir d'œufs de xénope [12]. Ce cytoplasme est obtenu en cassant les œufs par centrifugation à basse vitesse, en l'absence quasi complète de tampon. Il est possible de préparer des extraits interphasiques ou mitotiques à volonté, parce que les œufs de xénope sont pondus arrêtés en seconde métaphase de méiose et qu'il est possible de les faire passer aisément en interphase de façon synchrone à l'aide d'un choc électrique [13]. Des noyaux interphasiques ou des fuseaux mitotiques ont été reconstitués dans ces extraits acellulaires simplement en y ajoutant de l'ADN [14]. Cela se produit parce que les œufs de xénope contiennent, dans leur cytoplasme, toutes les protéines requises pour que les noyaux et les fuseaux nécessaires à l'accomplissement des 10 premiers cycles embryonnaires précoces s'assemblent pratiquement sans synthèse protéique. Il semble que ces extraits reproduisent relativement bien les conditions qui prévalent dans le cytoplasme de cellules intactes. En ajoutant des centrosomes à des extraits interphasiques ou mitotiques, il est donc possible d'étudier, in vitro, la dynamique des microtubules dans un environnement cytoplasmique défini reproduisant assez fidèlement ce qui se passe in vivo. En l'absence de chromosomes, les centrosomes ne forment pas de fuseaux, mais des asters. Cela permet de voir et d'analyser le comportement de microtubules individuels. Dans le cytoplasme interphasique, les centrosomes nucléent de très longs microtubules (plus de $20 \mu \mathrm{m}$ ) alors que dans le cytoplasme mitotique, ils nucléent des microtubules très courts (4-6 $\mu \mathrm{m})$ [15]. La dynamique fine de ces microtubules peut être analysée en ajoutant de la tubuline fluorescente et en examinant les microtubules en vidéo-microscopie [16]. Dans le cytoplasme interphasique, les paramètres de la dynamique des microtubules sont les suivants: $\mathrm{V}_{\mathrm{e}}=$ $10 \mu \mathrm{m} / \mathrm{min}, \mathrm{V}_{\mathrm{d}}=20 \mu \mathrm{m} / \mathrm{min}, \mathrm{f}_{\mathrm{cat}}=$
$0,01 / \mathrm{s}$ et $\mathrm{f}_{\mathrm{res}}=0,01 / \mathrm{s}$. Ces valeurs sont très proches de celles déterminées dans des cellules fibroblastiques en interphase. Dans le cytoplasme mitotique, on trouve à peu près les mêmes valeurs sauf pour $\mathrm{f}_{\text {cat }}$, qui est augmentée de 5 à 10 fois. Une analyse mathématique permettant de relier les paramètres de la dynamique des microtubules à leur longueur moyenne à l'équilibre a permis de démontrer que la petite taille des microtubules nucléés dans le cytoplasme mitotique est causée par l'augmentation de la fréquence de catastrophe [17]. On peut comprendre cela intuitivement. En effet, si chaque microtubule transite très fréquemment de la phase d'élongation à la phase de dépolymérisation, la probabilité pour que, dans une population de microtubules nucléés par un centrosome, un microtubule atteigne une grande longueur est très faible. Il existe donc un facteur dans le cytoplasme mitotique qui contrôle la longueur des microtubules en augmentant spécifiquement la valeur de $\mathrm{f}_{\text {cat }}$. La nature de ce facteur est encore inconnue. Il serait intéressant de déterminer s'il peut moduler la vitesse d'hydrolyse du GTP à l'extrémité des microtubules.

\section{La dynamique des microtubules est gouvernée par la kinase cycline B-cdc2}

Au cours des cinq dernières années, il a été montré que l'induction de la mitose dépend de l'activation périodique d'une protéine kinase. Cette enzyme est constituée d'une sousunité catalytique, le produit du gène cdc2 chez la levure Sacharomyces cerevisiae ou de ses homologues dans les cellules de vertébrés supérieurs, et d'une sous-unité régulatrice, la cycline B (l'homologue du produit du gène $c d c 13$ chez la levure) [18]. Apparemment, les premiers cycles du développement embryonnaire chez le xénope sont simplement entraînés par l'accumulation et la dégradation périodique de différentes cyclines mitotiques [19]. La sous-unité catalytique $\mathrm{p} 34^{\text {cdc2 }}$ est stockée en large excès dans les œufs, stable en quantité, mais activée seulement lorsqu'une concentration critique en cycline est atteinte. Lorsque l'activité kinase des complexes cycline/cdc2 


\section{RÉFÉRENCES}

30. Nicklas RB, Gordon GW. The total length of spindle microtubules depends on the number of chromosomes present. J Cell Biol 1985; 100 : 1-7.

31. Maro B, Howlett SK, Webb M. Nonspindle microtubule organizing centers in metaphase II-arrested mouse oocytes. J Cell Biol 1985 ; 101 : 1665-72.

32. Fernandez A, Brautigan DL, Lamb NJC. Protein phosphat ase type 1 in $\mathrm{mam}$ malian cell mitosis : chromosomal localization and involvement in mitotic exit. $J$ Cell Biol 1992; 116 : 1421-30

33. Enos AP, Morris NR. Mutation of a gene that encodes a kinesin-like protein blocks nuclear division in $A$. nidulans. Cell $1990 ; 60$ : 1019-27.

34. Endow SA, Henikoff SLN. Mediation of meiotic and carly mitotic chromosome segregation in Drosophila by a protein related to kinesin. Nature 1990 ; 345 : 81-3.

35. McDonald HB, Goldstein LSB. Identification and characterization of a gene coding for a kinesin-like protein in Drosophila. Cell 1990; 61 : 991-1000.

36. Hagan I, Yanagida M. Novel potential mitotic motor protein encoded by the fission yeast cut7 + gene. Nature $1990 ; 347$ : 563-6.

37. Hag an I, Yanagida M. Kinesin-related cut7 protein associates with mitotic and meiotic spindles in fission yeast. Nature 1992 ; 356 : 74-6.

38. Stewart RJ, Pesavento PA, Woerpel DN, Goldstein LS. Identification and partial characterization of six members of the kinesin superfamily in Drosophila. Proc Natl Acad Sci USA 1991; 88 : 8470-4.

39. Steuer ER, Wordeman L, Schroer TA, Sheetz MP. Localization of cytoplasmic dynein to mitotic spindles and kinetochores. Nature 1990 ; 345 : 266-8.

40. Pfarr CM, Coue M, Grissom PM, Hays TS, Porter ME, McIntosh JR. Cytoplasmic dynein is localized to kinetochores during mitosis. Nature $1990 ; 345$ : 263-5.

41. Hyman AA, Mitchison T. Two different microtubule-based motor activities with opposite polarities in kinetochores. Nature 1991; 351: 206-11.

42. Bailly E, Dorée M, Nurse P, Bornens M. p34cdc2 is located in both nucleus and cytoplasm; part is centrosomally associated at $\mathrm{G} 2 / \mathrm{M}$ and enters vesicles at an aphase. EMBO J $1989 ; 8$ : 3985-95. atteint un seuil critique, le mécanisme de dégradation des cyclines mitotiques est mis en route, les cyclines dégradées et la kinase inactivée [20]. Ce processus rend compte, dans ces embryons, de l'oscillation rapide du cycle entre interphase et mitose toutes les 30 minutes. Les extraits cytoplasmiques préparés à partir d'œufs interphasiques utilisés pour étudier la dynamique des microtubules contiennent $\mathrm{p} 34^{\mathrm{cdc} 2}$ sous une forme inactive, mais ne contiennent pas de cyclines $[21,22]$. On peut donc rajouter à ces extraits soit la kinase cycline B-cdc2 purifiée sous sa forme active [23], soit une cycline qui, en se combinant aux molécules de p34 ${ }^{\text {cdc2 }}$ présentes dans l'extrait, les active [24, 25]. Dans les deux cas, la dynamique des microtubules passe d'un régime interphasique au régime mitotique $[15,17]$. Par conséquent, le changement de dynamique et donc de longueur des microtubules lors de l'entrée en mitose est dû à un événement de phosphorylation qui dépend de la kinase $\mathrm{cdc} 2$. Il reste à déterminer la nature des facteurs impliqués dans le contrôle de la dynamique des microtubules et si la kinase cycline B-cdc2 agit directement ou indirectement sur eux.

Orientation préférentielle des microtubules vers les chromosomes en métaphase

Lors de l'entrée en métaphase, les microtubules qui polymérisent vers les chromosomes sont beaucoup plus longs que les autres. De plus, en général, un fuseau mitotique ne se forme pas en l'absence de chromosomes. Par exemple, si l'on ajoute un grand nombre de centrosomes dans un extrait cytoplasmique mitotique, chaque centrosome produit un petit aster, mais même si les microtubules de deux asters entrent en contact, ils n'interagissent pas. En revanche, si l'on rajoute des chromosomes au même extrait, un fuseau se forme [26]. L'orientation préférentielle des microtubules vers les chromosomes pourrait être simplement due à leur capture suivie de stabilisation par les kinétochores [3, 27]. Cependant, les kinétochores ne semblent pas être essentiels pour l'assemblage du fuseau [26]. Il semble que la chromatine mitotique ait un effet local de stabi- lisation sur les microtubules [28]. Cette stabilisation n'implique pas une interaction physique des microtubules avec le chromosome. Elle semble être due à une action des chromosomes sur le cytoplasme environnant. En fait, il y a une corrélation entre la taille du fuseau et la masse de chromatine ou le nombre de chromosomes [29-31]. La nature biochimique de cet effet est encore mal définie, mais ce que nous avons appris du contrôle de la dynamique des microtubules par la kinase cdc2 suggère une explication.

Nous savons que, en métaphase, la longueur moyenne des microtubules nucléés par des centrosomes a une valeur bien définie qui est déterminée, la valeur de $f_{\text {cat }}$. Cette fréquence de catastrophe est elle-même contrôlée par un facteur dont l'activité augmente lorsqu'il est phosphorylé directement ou indirectement par la kinase cdc2. Toute protéine phosphorylée par une kinase est déphosphorylée par une phosphatase. Donc, à tout moment, la proportion de la forme phosphorylée d'une protéine dépend de l'activité relative de la kinase qui ajoute et de la phosphatase qui enlève le phosphate sur le site de phosphorylation (figure $3 a$ ). Dans les extraits de xénope, la longueur des microtubules nucléés par des centrosomes peut être manipulée en ajoutant différentes quantités de kinase cdc2 (figure $3 a$ ). Plus le niveau de l'activité kinase est élevée, plus les microtubules sont courts. En revanche, si l'on ajoute une phosphatase en excès à un extrait contenant un haut niveau de kinase cdc2, la longueur des microtubules augmente (figure $3 a$ ). Cela signifie que $\mathrm{f}_{\text {cat }}$ et la longueur moyenne des microtubules sont déterminés, en mitose, par l'activité relative de la kinase cdc2 et d'une phosphatase qui s'oppose à l'effet de la kinase [21].

Si le niveau d'activité de la phosphatase est élevé dans les chromosomes par rapport au reste du cytoplasme, la fréquence de catastrophes sera réduite autour des chromosomes. Par conséquent, les microtubules polymérisant vers les chromosomes seront plus longs et auront une durée de vie suffisante pour interagir avec les kinétochores ou d'autres microtubules provenant du pôle opposé (figure $3 b$ ). 
Une phosphatase de type 1 est effectivement fortement enrichie dans les chromosomes mitotiques [32]. Ce modèle est à présent hypothétique, mais l'idée est intéressante parce qu'il s'agit d'un processus qui, s'il est démontré, pourrait avoir une implication générale. En effet, par la localisation de kinases et de phosphatases dans l'espace cellulaire, des gradients de phosphorylation de substrats spécifiques peuvent être établis, engendrant des asymétries fonctionnelles et structurales.

\section{Moteurs protéiques et fuseau mitotique}

Il est clair, en observant la figure 1, que des protéines spécialisées doivent être impliquées dans l'interaction des microtubules antiparallèles dans la région équatoriale du fuseau ainsi
Activité de la kinase cdc2 dans l'extrait

$4 u / \mu l$

\section{Asters de microtubules nucléés}
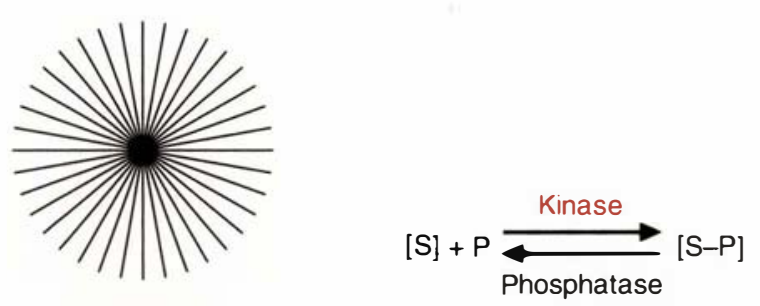

$8 \mathrm{u} / \mu \mathrm{l}$

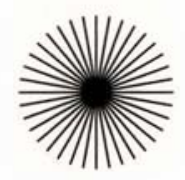

:

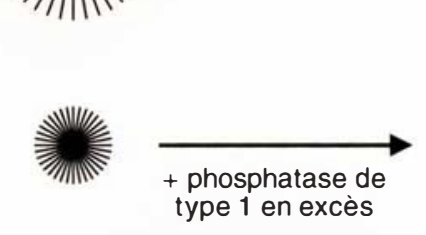

A type 1 en excès

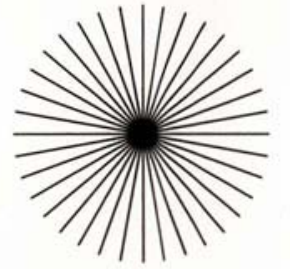

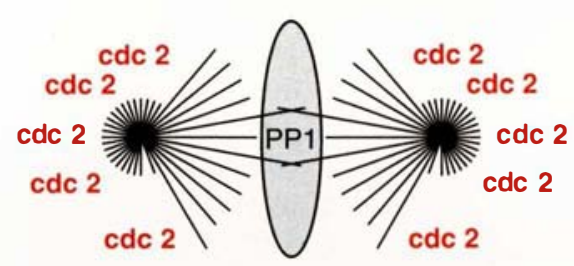

Figure 3. Dans un cytoplasme mitotique, la longueur des microtubules est déterminée par l'activité relative de la kinase cdc2 et d'une phosphatase. A. Des quantités croissantes de kinase ont été ajoutées à un extrait d'œufs de xénope interphasiques. L'activité kinase mesurée dans l'extrait est indiquée à gauche (en utilisant I'histone $\mathrm{H} 1$ comme substrat) et la longueur des microtubules nucléés dans l'extrait par des centrosomes, schématisée à droite. L'addition d'une phosphatase de type 1 à l'extrait contenant une forte activité kinase, et donc des microtubules courts, conduit à un rallongement des microtubules (en bas). L'équation de droite montre comment la concentration en substrat phosphorylé peut être contrôlée par un couple phosphatase/kinase. B. Mécanisme hypothétique, fondé sur les résultats ci-dessus, de l'orientation préférentielle des microtubules vers les chromosomes en mitose. Un chromosome est schématisé entre deux asters. II contient une forte concentration d'une phosphatase (type 1 probablement) (PP1) qui diminue localement autour des chromosomes la concentration en facteur phosphorylé augmentant la fréquence de catastrophes. Cela permet aux microtubules de s'allonger préférentiellement vers les chromosomes.

$\mathrm{m} / \mathrm{s} n^{\circ} 2$ vol. 9, féurier 93 que dans l'interaction microtubuleskinétochores. Il est également clair que ces interactions doivent être dynamiques. L'implication de moteurs protéiques dans l'assemblage et la fonction du fuseau a été récemment mise en évidence par des méthodes de génétique moléculaire et des méthodes immunocytochimiques ainsi qu'à l'aide de tests de mobilité réalisés in vitro en vidéo-microscopie.

Moteurs moléculaires et assemblage du fuseau

L'évidence la plus directe d'une implication de protéines ressemblant à la kinésine dans la formation du fuseau mitotique est venue de la génétique moléculaire. En effet, une série de mutations connues pour affecter la morphologie des fuseaux mitotiques et méiotiques, ou la ségrégation des chromosomes, sc sont avérées être localisées au niveau des gènes codant pour des molécules ayant une forte homologie avec la kinésine, en particulier dans la région motrice de ces molécules (figure 4 , p. 138) [33-36]. Les phénotypes les plus marqués ont été obtenus avec les mutants bimC chez A. nidulans et cut 7 chez $S$. pombe. Dans les deux mutants, l'interdigitation des microtubules qui se produit normalement dans la zone équatoriale du fuseau n'apparaît pas. Seulement deux demifuseaux se forment. L'analyse immunocytochimique a montré que, dans les levures sauvages, la protéine est localisée à la fois près des pôles et le long des microtubules du fuseau, alors que dans les levures mutantes, on ne la retrouve que près des pôles [37]. Comme il s'agit d'une protéine de type kinésine, on peut faire l'hypothèse qu'elle se déplace vers l'extrémité + des microtubules, c'està-dire du pôle vers l'équateur du fuseau. Si c'est effectivement le cas, le résultat obtenu en immunocytochimie est très intéressant. En effet, il suggère que la mutation empêche la protéine de migrer normalement le long des microtubules. Puisque l'interdigitation ne se produit pas, il est probable que cette protéine est impliquée dans ce processus en glissant entre deux microtubules de polarité opposée vers l'équateur du fuseau (voir figure 1). Malheureusement, on ne sait pas encore si Cut7 se déplace 
vers l'extrémité + ou - des microtubules. Il faut donc prendre cette interprétation avec réserve car une autre protéine impliquée dans la ségrégation des chromosomes chez la drosophile (Ncd) appartient également à la famille des protéines reliées à la kinésine tout en ayant une polarité de mouvement opposée [6]. De nombreuses molécules de ce type sont en cours de caractérisation [38] et de nouveaux tests in vitro sont en développement. Il est donc clair que, dans un avenir assez proche, nous en saurons beaucoup plus sur la façon dont l'interdigitation des microtubules se produit dans le fuseau.

Le kinétochore contient des moteurs dont la polarité peut être inversée par phosphorylation

En prophase, le mouvement des chro- mosomes vers les pôles se produit à une vitesse correspondant à peu près à la vitesse de déplacement des molécules de dinéine le long des microtubules. De plus, ce mouvement commence par une migration vers l'extrémité - des microtubules. Récemment, deux groupes ont mis en évidence la présence de dinéine dans le fuseau, au niveau des kinétochores et sur les microtubules, plus particulièrement près des pôles, par des méthodes immunocytochimiques [39, 40]. Afin de mettre en évidence de façon définitive la présence de moteurs dans les kinétochores, Hyman et Mitchison [41], ont développé un test de mobilité permettant de suivre le mouvement des microtubules le long des kinétochores. Le principe du test est décrit dans la figure 5. Des chromosomes mitotiques

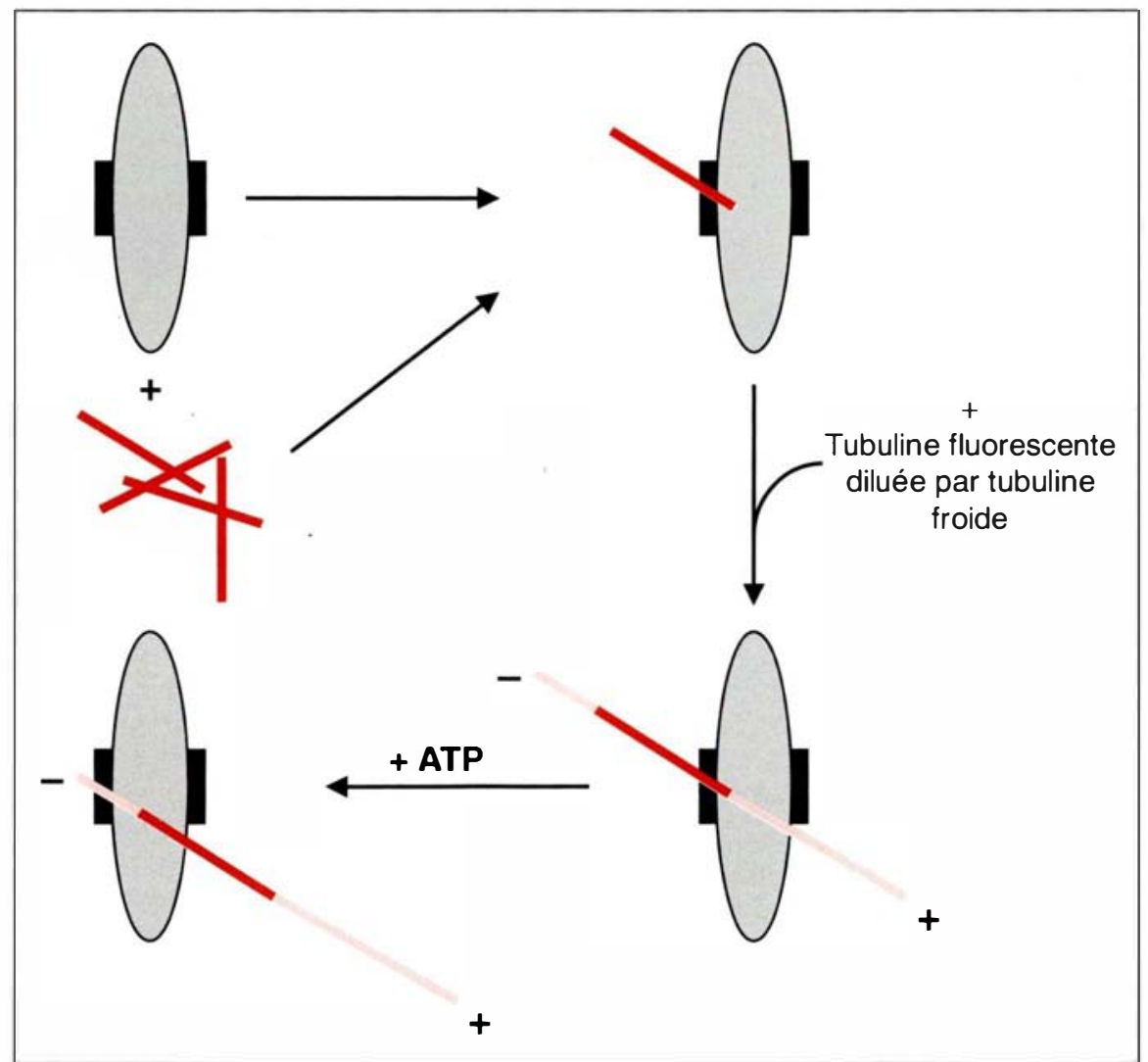

Figure 4. Test de mobilité des microtubules le long des kinétochores (rectangles sur les chromosomes). Des microtobules courts fortement marqués avec un traceur fluorescent (barres rouges) sont stabilisés et ajoutés à des chromosomes entre lame et lamelle. Dans ces conditions, ils se fixent aux kinétochores. Ensuite, des sous-unités de tubuline peu fluorescentes sont rajoutées. Ces molécules forment des microtubules peu fluorescents (en rose) aux extrémités + et - des microtubules stables accrochés aux kinétochores, permettant de visualiser leur polarité. Lorsque l'on rajoute de l'ATP, les microtu- sont préparés à partir de cellules en culture, puis incubés entre lame et lamelle en présence de courts segments de microtubules brillamment marqués par un traceur fluorescent. Ces microtubules sont capturés par les kinétochores. Ensuite, de la tubuline marquée avec le même traceur, mais diluée avec de la tubuline non marquée, est ajoutée. Cette tubuline forme donc des microbubules moins brillants. Comme la tubuline polymérise plus rapidement à l'extrémité + des fragments fortement marqués, cette technique permet d'engendrer des microtubules dont la polarité est visible. Ensuite, de l'ATP est ajouté. Immédiatement, les microtubules commencent à glisser rapidement le long du kinétochore (20-30 $\mu \mathrm{m} / \mathrm{min})$, l'extrémité + vers l'avant. Il y a donc bien un moteur dépendant de l'ATP qui se déplace vers l'extrémité - des microtubules dans le kinétochore. En utilisant le même test, ils ont également montré qu'après phosphorylation, la direction du mouvement s'inverse. Dans ce mode de fonctionnement du moteur, le déplacement des microtubules est également environ 10 fois plus lent.

Il est donc probable que le mouvement des chromosomes le long des microtubules implique deux types de moteurs et que l'activité relative de chaque moteur est contrôlée par phosphorylation. En conjonction avec les résultats obtenus sur le contrôle par phosphorylation de la dynamique des microtubules décrits plus haut, ces résultats nous fournissent peut être la clef du mécanisme de positionnement des chromosomes sur la plaque métaphasique. J'ai déjà discuté comment un gradient de phosphorylation d'un substrat donné peut être engendré par un couple phosphatase/kinase, si les deux enzymes sont localisées dans la cellule. Comme dans le cas de la dynamique des microtubules, il est possible que l'activité des deux types de moteurs soit précisément ajustée par leur niveau de phosphorylation. Si les kinasẹs et les phosphatases qui contrôlent cette phosphorylation sont localisées, l'une aux pôles par exemple et l'autre sur les chromosomes, on peut comprendre comment les chromosomes sont positionnés. En fait, la kinase cycline B-cdc2 est for- 


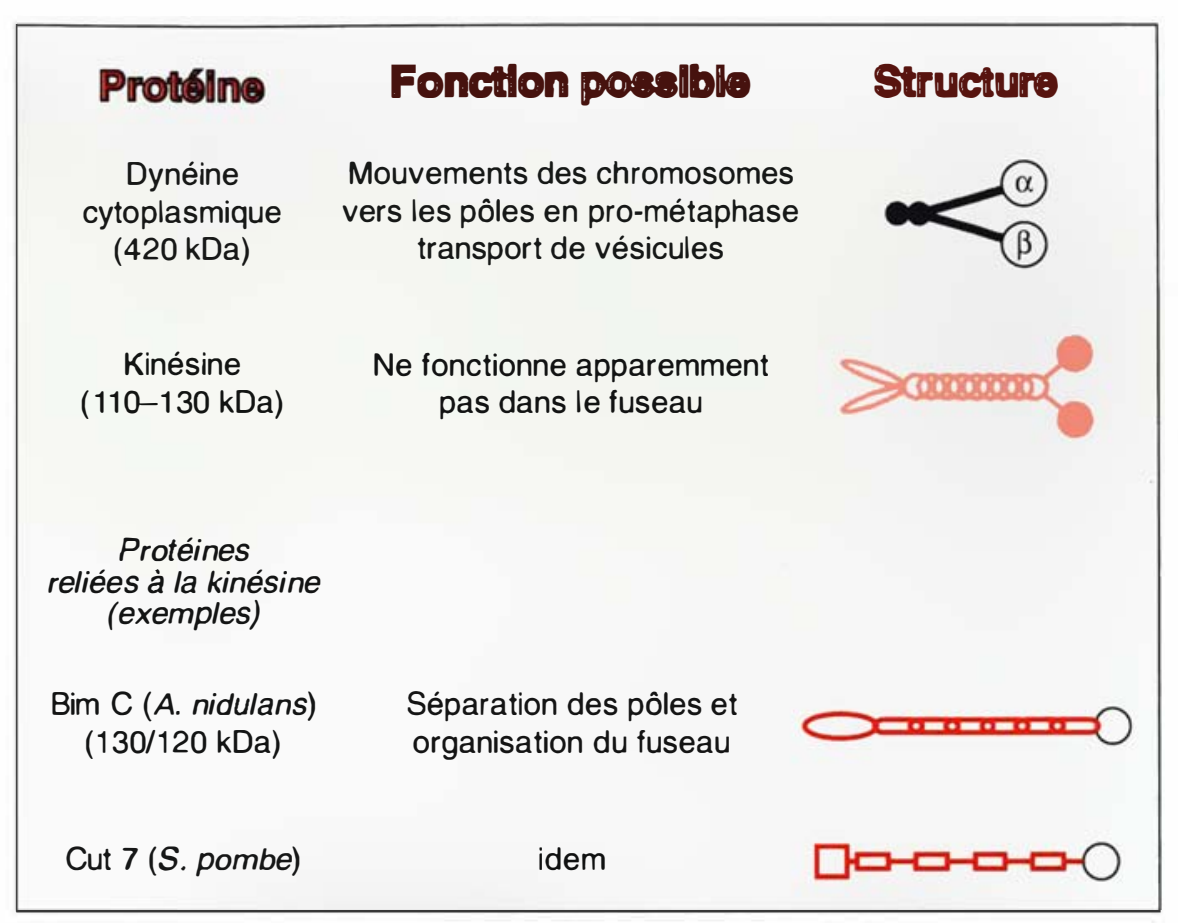

Figure 5. Différences et similarités entre les moteurs impliqués dans I'assemblage et la fonction du fuseau mitotique. II s'agit d'exemples, non d'une liste exhaustive. La dinéine se déplace vers l'extrémité - des microtubules. Les autres molécules indiquées, vers l'extrémité +. Le domaine moteur est symbolisé par un cercle dans chaque cas, il est très conservé entre les différentes molécules de kinésine. Le fait que la kinésine ne fonctionne pas dans le fuseau alors que des molécules qui lui sont reliées, comme BimC et Cut 7 , sont essentielles suggère que la région non motrice est importante pour la spécificité de fonction de ces moteurs.

tement concentrée aux pôles du fuseau [42], et une phosphatase spécifique (phosphatase 1) est concentrée dans les chromosomes [32]. Ce couple pourrait contrôler à la fois la fréquence de catastrophe et l'activité relative des deux moteurs présents dans les kinétochores. Cela est encore spéculatif, mais ces expériences commencent à nous indiquer la direction dans laquelle il faut chercher pour comprendre comment la biochimie peut produire des structures aussi belles et évanescentes que le fuseau mitotique.

\section{Conclusion}

L'assemblage du fuseau mitotique fait intervenir une coordination précise de la dynamique des microtubules avec l'activité de moteurs moléculaires. Cette coordination est, très probable- du mécanisme d'assemblage du fuseau mitotique a d'ores et déjà considérablement progressé

\section{Summary}

Towards a molecular description of mitotic spindle assembly

Morphogenesis of the mitotic spindle raises original questions because it is a transient, highly dynamic structure. The reorganization of microtubules from a radial array in interphase into a bipolar spindle during mitosis, results from phosphorylation events induced by the cyclin Bcdc2 kinase. These phosphorylations control microtubule dynamics and probably the activity of motor proteins essential in spindle assembly. At the onset of mitosis, microtubules become extremely dynamic and shorten dramatically. Their preferential orientation towards the chromosomes seems to result from a local effect of chromatin on the surrounding cytoplasm that stabilizes microtubules. Spindle bipolarity is established and maintained by interactions between anti-parallel microtubules in the equatorial area of the spindle. These interactions seem to involve motor proteins belonging to the kinesin superfamily. During their positioning on the metaphase plate, chromosomes move along microtubules. These movements are also due to motor proteins (probably belonging to both the kinesin and dynein families of proteins) localized in the kinetochores. Their directionality of movement along the microtubule lattice is regulated by phosphorylation suggesting a mechanism by which the chromosomes are positioned on the metaphase plate.

\section{TIRÉS A PART}

E. Karsenti. 